\title{
Maximizing light emission from silicon nanocrystals - The role of hydrogen
}

\author{
A.R. Wilkinson, R.G. Elliman * \\ Department of Electronic Materials Engineering, Research School of Physical Sciences and Engineering, \\ The Australian National University, Canberra, ACT 0200, Australia
}

Available online 16 September 2005

\begin{abstract}
Time-resolved photoluminescence measurements are undertaken to determine the passivation kinetics of luminescence-quenching defects during isothermal and isochronal annealing in molecular and atomic hydrogen. The latter employs an alneal process in which atomic hydrogen is generated by reactions between a deposited $\mathrm{Al}$ layer and $\mathrm{H}_{2} \mathrm{O}$ or $-\mathrm{OH}$ radicals in the $\mathrm{SiO}_{2}$. Passivation and desorption kinetics are shown to be consistent with the existence of two classes of non-radiative defects: one that reacts with either atomic or molecular hydrogen, and the other that reacts only with atomic hydrogen.
\end{abstract}

(C) 2005 Elsevier B.V. All rights reserved.

PACS: $81.07 . \mathrm{Bc} ;$ 73.63.Bd; 78.67.Bf

Keywords: Nanocrystal; Silicon; Passivation; Hydrogen; Luminescence; Dangling-bond

\section{Introduction}

A major limitation to the integration of electronic and optical functionality using standard silicon processing technology is the lack of an efficient silicon-based light source, such as a light-emitting diode or laser. Consequently, the discovery in the early 1990s of efficient room-temperature photoluminescence (PL) in porous [1] and nanocrystalline [2] silicon stimulated considerable interest and subsequent research effort $[3,4]$. Although the exact mechanism for light emission still remains controversial, it is well established that the emission energy is dependent on nanocrystal size [5] and that non-radiative surface defects (including $P_{\mathrm{b}}$-type defects) compete with radiative processes [6].

Silicon nanocrystals embedded in $\mathrm{SiO}_{2}$ exhibit strong room temperature luminescence and it is well known that both the luminescence intensity and lifetime can be increased by annealing in molecular hydrogen [7-10]. This

\footnotetext{
* Corresponding author. Tel.: +61 26125 0521; fax: +61 261250511.

E-mail address: rob.elliman@anu.edu.au (R.G. Elliman).
}

is attributed to hydrogen passivation of competing nonradiative defect centers, such as $\mathrm{Si}$ dangling bonds located at the nanocrystal surface. The class of dangling bond defects known as $P_{\mathrm{b}}$ centers is thought to be the dominant recombination site [10] and it has been shown that one such defect is sufficient to quench the visible luminescence of a $\mathrm{Si}$ nanocrystal [6].

Passivation is usually achieved by thermal annealing of the nanocrystals in forming gas. However, annealing in atomic hydrogen is known to result in more effective passivation for planar $\mathrm{Si} / \mathrm{SiO}_{2}$ interfaces, with defect densities in the order of $10^{10} \mathrm{~cm}^{-2} \mathrm{eV}^{-1}$ achievable [11]. Moreover, significant concentrations of atomic hydrogen are known to be generated within an oxide layer if a chemically active metal such as $\mathrm{Al}$ is deposited on the sample surface prior to annealing $[12,13]$. When $\mathrm{Al}$ is used, this process is sometimes known as an alneal. The alneal is used extensively in solar cell fabrication to reduce the surface recombination rate [14]. The hydrogen is believed to be generated by the reaction of $\mathrm{Al}$ with water vapor absorbed at the $\mathrm{SiO}_{2}$ surface or $-\mathrm{OH}$ radicals present in the $\mathrm{SiO}_{2}$ [15]. 


\section{Experimental}

Thermally grown $\mathrm{SiO}_{2}$ layers of $1.25 \mu \mathrm{m}$ thickness were implanted with $400 \mathrm{keV} \mathrm{Si}^{+}$to a fluence of $2 \times 10^{17} \mathrm{~cm}^{-2}$ at room-temperature, producing a peak excess $\mathrm{Si}$ concentration of 10 at. $\%$ at a mean projected range of $630 \mathrm{~nm}$, calculated using SRIM [16]. Nucleation and growth of nanocrystals was achieved by annealing the implanted samples in a quartz-tube furnace at $1100{ }^{\circ} \mathrm{C}$ for $1 \mathrm{~h}$ in flowing Ar.

Molecular hydrogen passivation was achieved by thermal annealing in high-purity forming gas $\left(5 \% \mathrm{H}_{2}\right.$ in $\left.\mathrm{N}_{2}\right)$. A rapid thermal processor (AET Thermal RX) was used for annealing times $\leqslant 10 \mathrm{~min}$, with longer anneals performed in a conventional tube furnace. The alneal process consisted of evaporating a $100 \mathrm{~nm}$ layer of $\mathrm{Al}$ onto the oxide surface prior to thermal annealing in $\mathrm{N}_{2}$ or forming gas. The Al layer was subsequently removed by etching in $90{ }^{\circ} \mathrm{C} \mathrm{H}_{3} \mathrm{PO}_{4}(85 \%)$ for $1 \mathrm{~min}$. Hydrogen desorption was performed in a $\mathrm{N}_{2}$ environment. The samples used were annealed for $1 \mathrm{~h}$ in $\mathrm{N}_{2}$ at $1100{ }^{\circ} \mathrm{C}$, to form the nanocrystals. The samples were then passivated with either $\mathrm{H}_{2}$ or $\mathrm{H}$.

Photoluminescence (PL) measurements were performed at room temperature, using the $532 \mathrm{~nm}$ line of a diodepumped solid state laser as the excitation source. Emitted light was analyzed using a single-grating monochromator (TRIAX-320) and detected with a liquid-nitrogen cooled CCD array (EEV CCD30-11).

Time-resolved PL measurements were performed by modulating the laser beam with an acousto-optic modulator (Brimrose TEM-85-10). A room-temperature multialkali photomultiplier (Hamamatsu R928) was used to detect the light from the exit port of the monochromator with the grating centered at $800 \mathrm{~nm}$ (band pass of $40 \mathrm{~nm}$ ). The signal from the photomultiplier was collected using a digital storage oscilloscope. The timing resolution of the system is $<1 \mu \mathrm{s}$.

\section{Results and discussion}

The effect of hydrogen on nanocrystal PL is demonstrated in Fig. 1, which compares spectra from an unpassivated sample and samples passivated with molecular (1 and $16 \mathrm{~h}$ ) and atomic hydrogen. The data illustrate two important points: (a) that the luminescence intensity can be enhanced by more than one order-of-magnitude by annealing in molecular hydrogen and (b) that further significant enhancement is possible by exposure to atomic hydrogen. The latter could result from differences in the reaction rates of $\mathrm{H}_{2}$ and $\mathrm{H}$ with the non-radiative defects. However, it has previously been shown that a $1 \mathrm{~h}$ anneal at $500{ }^{\circ} \mathrm{C}$ produces optimal passivation for samples annealed in forming gas [10] and that this passivation level is unchanged during longer anneals up to $16 \mathrm{~h}$, as shown in Fig. 2. This suggests that the system is in a quasi-thermal equilibrium state with respect to the $\mathrm{H}_{2}$ reaction. The additional effect of atomic hydrogen therefore suggests

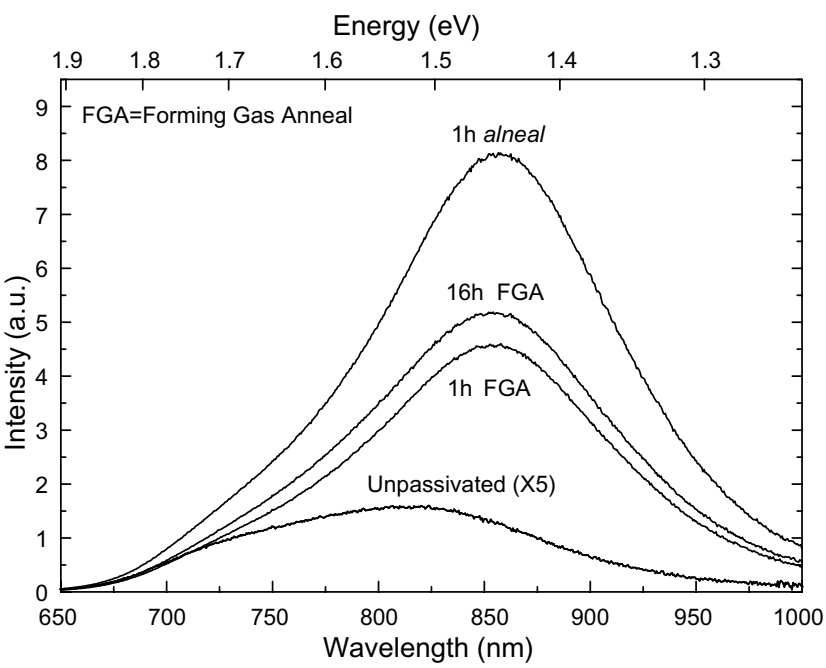

Fig. 1. Room-temperature photoluminescence spectra from an unpassivated sample, a sample passivated in forming gas for $1 \mathrm{~h}$ at $500{ }^{\circ} \mathrm{C}$, and a sample annealed in $\mathrm{N}_{2}$ for $1 \mathrm{~h}$ at $500{ }^{\circ} \mathrm{C}$ after the evaporation of an $\mathrm{Al}$ layer. The spectrum for a sample annealed in forming gas for $16 \mathrm{~h}$ at $500{ }^{\circ} \mathrm{C}$ is also included for comparison.

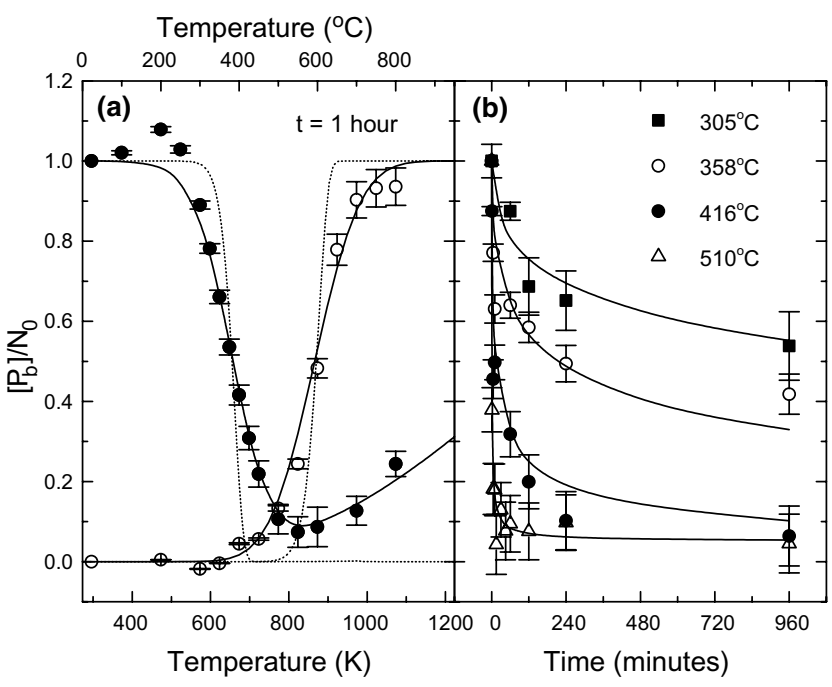

Fig. 2. Plots of relative $P_{\mathrm{b}}$ defect concentration versus (a) annealing temperature of hydrogen passivation $(O)$ and dissociation $(\bigcirc)$ and $(b)$ annealing time, during hydrogen passivation at $305^{\circ} \mathrm{C}, 358^{\circ} \mathrm{C}, 416^{\circ} \mathrm{C}$ and $510^{\circ} \mathrm{C}$. The solid lines represent the global least-squares fit of the GST model to experimental data derived from time-resolved PL.

the presence of a different subset of non-radiative defects. Specifically, defects that act as recombination centers but do not react with molecular hydrogen.

The passivation process is reversible, such that annealing of passivated samples in a hydrogen-free environment can be used to desorb hydrogen from the nanocrystals. This is believed to proceed via thermal dissociation of $\mathrm{Si}-\mathrm{H}$ bonds and has the effect of reducing the nanocrystal luminescence to pre-passivation levels. The kinetics of the passivation and desorption processes provide insight into the chemical reactions responsible for these processes. Such 
a study has recently been undertaken for passivation in molecular hydrogen [10], as summarized in Fig. 2.

This figure shows the relative concentration of defects that can be passivated by molecular hydrogen, as a function of annealing temperature, for both the passivation and desorption reactions. Also shown is the temporal dependence of the passivation reaction (Fig. 2(b)). The solid lines show a fit of the generalised simple thermal (GST) model developed by Stesmans $[17,18]$ for $\mathrm{Si} / \mathrm{SiO}_{2}$ planar interfaces. The model includes a Gaussian distribution of activation energies for the passivation and desorption reactions in order to account for variable defect configurations due to stress. (The assumption of single activation energies, consistent with Brower's model $[19,20]$ leads to a less adequate fit as illustrated by the dotted lines in Fig. 2(a).)

Although a full kinetic analysis of the atomic hydrogen reactions has yet to be completed, a comparison of the depassivation rate for samples passivated in molecular and atomic hydrogen suggests that hydrogen desorbs from the sites passivated by atomic hydrogen more readily than from those passivated by molecular hydrogen. This is illustrated by the data of Fig. 3. For example, after annealing at $525^{\circ} \mathrm{C}$ for $1 \mathrm{~h}$, the PL intensity from samples passivated in atomic and molecular hydrogen have dropped to a similar level, even though the initial intensity was much higher in the atomic hydrogen case.

This difference is further highlighted in Fig. 4, which shows the normalized number of emitting nanocrystals as a function of temperature during desorption of hydrogen from samples initially passivated in atomic and molecular hydrogen. This quantity is derived from the simple relation, valid in the low pump regime, $n^{*} \propto \frac{I}{\tau}$, where $n^{*}$ is the number of emitting nanocrystals, and $I$ and $\tau$ are the PL intensity and lifetime, respectively (in this case measured at $\lambda=800 \mathrm{~nm}$ ). On the assumption that atomic

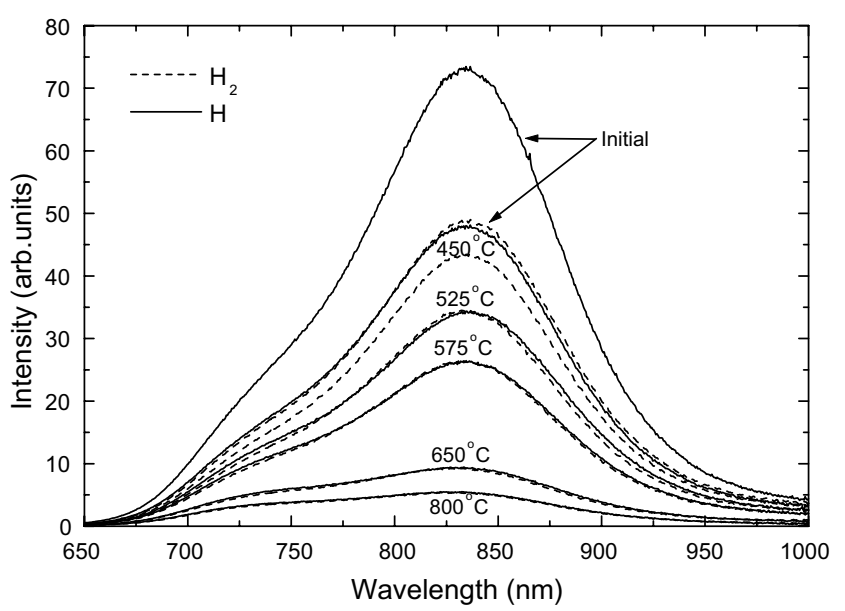

Fig. 3. Room-temperature photoluminescence spectra showing decrease in intensity due to desorption of hydrogen by annealing for $1 \mathrm{~h}$ in $\mathrm{N}_{2}$ at the temperatures indicated on the graph. $\mathrm{H}_{2}$ refers to samples initially annealed in forming gas for $1 \mathrm{~h}$ at $500{ }^{\circ} \mathrm{C}$ and $\mathrm{H}$ refers to samples initially annealed in $\mathrm{N}_{2}$ for $30 \mathrm{~min}$ at $400{ }^{\circ} \mathrm{C}$ after $\mathrm{Al}$ metallization. NB: There are two curves for each temperature.

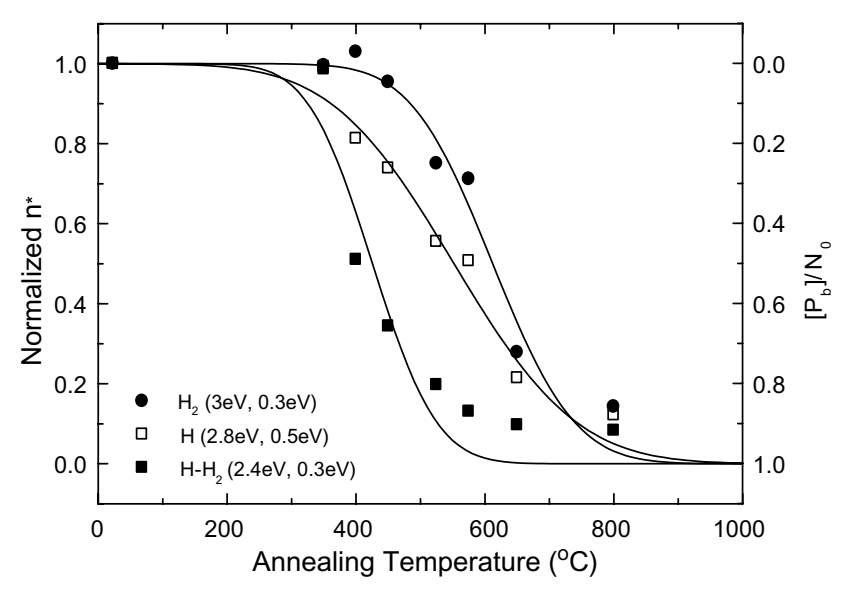

Fig. 4. Normalized number of emitting nanocrystals, derived from timeresolved PL measurements on samples shown in Fig. 3, along with more desorption temperatures. The solid lines are fits of the GST model. The desorption activation energy and spread in activation energy for each curve are indicated on the graph.

hydrogen passivates both the defects passivated by molecular hydrogen as well as a second class of defects that do not react with molecular hydrogen, the difference between these two curves (before normalization) can be used to isolate the latter contribution. Such analysis is included in Fig. 4, together with modeling of the two contributions that give desorption activation energies of $3 \mathrm{eV}$ and $2.4 \mathrm{eV}$ for the two defect subsets. The pre-exponential factor for the dissociation rate constant was fixed at $2 \times 10^{13} \mathrm{~s}^{-1}$ for these fits.

Earlier studies have revealed a correlation between the concentration of ESR active $P_{\mathrm{b}}$ defects and molecular hydrogen passivation suggesting that this class of defect is a dominant non-radiative recombination center [21]. However, the results of the present study show that annealing in atomic hydrogen can significantly increase the level of passivation above that achievable with molecular hydrogen. This observation, along with the differences in desorption kinetics (Figs. 3 and 4), supports a model in which more than one class of non-radiative defect is active. Indeed, such a scenario is consistent with work by Gheorghita et al. [22] that provided alternative evidence for two classes of defects at the (111) $\mathrm{Si} / \mathrm{SiO}_{2}$ interface in addition to the $P_{\mathrm{b}}$ defect: one that can be passivated with $\mathrm{H}_{2}$, and one that cannot.

\section{Conclusions}

Time-resolved photoluminescence was used to study the passivation and desorption kinetics of non-radiative defects in silicon nanocrystals annealed in molecular and atomic hydrogen. The reaction kinetics for samples annealed in molecular hydrogen were found to be well described by the generalized simple thermal (GST) model proposed by Stesmans for planar $\mathrm{Si} / \mathrm{SiO}_{2}$ interfaces [17]. However, annealing in atomic hydrogen was shown to result in greater levels of passivation than were achievable 
with molecular hydrogen under optimal conditions. This suggested the presence of at least two classes of non-radiative defect: one that reacts with either atomic or molecular hydrogen, and a second that reacts only with atomic hydrogen. This interpretation was supported by hydrogen desorption data that showed a lower activation energy for desorption from sites passivated with atomic hydrogen $\left(E_{\mathrm{a}}=2.4 \mathrm{eV}\right)$ than for those passivated with molecular hydrogen $\left(E_{\mathrm{a}}=3.0 \mathrm{eV}\right)$.

\section{Acknowledgement}

The authors acknowledge financial support from the Australian Research Council Discovery Program.

\section{References}

[1] L.T. Canham, Appl. Phys. Lett. 57 (1990) 1046.

[2] H. Takagi, H. Ogawa, Y. Yamazaki, A. Ishizaki, T. Nakagiri, Appl. Phys. Lett. 56 (1990) 2379.

[3] L. Pavesi, J. Phys.: Condens. Matter 15 (2003) R1169.

[4] D. Kovalev, H. Heckler, G. Polisski, F. Koch, Phys. Status Solidi B 215 (1999) 871
[5] G. Ledoux, O. Guillois, D. Porterat, C. Reynaud, F. Huisken, B. Kohn, V. Paillard, Phys. Rev. B 62 (2000) 15942.

[6] M. Lannoo, C. Delerue, G. Allan, J. Lumin. 70 (1996) 170.

[7] K.S. Min, K.V. Shcheglov, C.M. Yang, H.A. Atwater, M.L. Brongersma, A. Polman, Appl. Phys. Lett. 69 (1996) 2033.

[8] E. Neufield, S. Wang, R. Apetz, C. Buchal, R. Carius, C.W. White, D.K. Thomas, Thin Solid Films 294 (1997) 238.

[9] S.P. Withrow, C.W. White, A. Meldrum, J.D. Budai, D.M. Hembree Jr., J.C. Barbour, J. Appl. Phys. 86 (1999) 396.

[10] A.R. Wilkinson, R.G. Elliman, Phys. Rev. B 68 (2003) 155302.

[11] S.K. Ghandi, VLSI Fabrication Principles: Silicon and Gallium Arsenide, second ed., Wiley, NY, 1994

[12] R.F. Pierret, Field Effect Devices, second ed., Addison-Wesley, Reading, MA, 1990.

[13] A.R. Wilkinson, R.G. Elliman, Appl. Phys. Lett. 83 (2003) 5512.

[14] M.J. Kerr, A. Cuevas, Semicond. Sci. Technol. 17 (2002) 35.

[15] M.L. Reed, J.D. Plummer, J. Appl. Phys. 63 (1988) 5776.

[16] J.F. Ziegler, J.P. Biersack, U. Littmark, The Stopping and Range of Ions in Solids, Pergamon Press, New York, 1985.

[17] A. Stesmans, J. Appl. Phys. 88 (2000) 489.

[18] A. Stesmans, J. Appl. Phys. 92 (2002) 1317.

[19] K.L. Brower, Phys. Rev. B 38 (1988) 9657.

[20] K.L. Brower, Phys. Rev. B 42 (1990) 3444.

[21] M. López, B. Garrido, C. García, P. Pellegrino, A. Pérez-Rodríguez, J. Morante, C. Bonafos, M. Carrada, A. Claverie, Appl. Phys. Lett. 80 (2002) 1637.

[22] L. Gheorghita, E. Ogryzlo, J. Appl. Phys. 87 (2000) 7999. 